\title{
Propondo uma ferramenta tecnológica para comunicação entre enfermeiro e paciente em oncologia
}

\begin{abstract}
Fabiane de Amorim Almeida1 e Mahyra Medeiros Vieira²
${ }^{1}$ Faculdade Israelita de Ciências da Saúde Albert Einstein, Brasil |

fabi.almeida17@outlook.com; https://orcid.org/0000-0002-8062-3579

${ }^{2}$ Hospital Israelita Albert Einstein, Brasil | mahyra.vieira@einstein.br | http://orcid.org/00000002-4874-68X

Resumo: Introdução: A presença do enfermeiro navegador nos cenários de cuidado aos pacientes em oncologia tem se tornado uma tendência no Brasil e no mundo, com destaque para o câncer colorretal. Uma das principais estratégias de abordagem utilizadas por estes profissionais é a comunicação, que tem sido facilitada com o uso da tecnologia. Objetivos: Compreender as expectativas dos pacientes com tumores colorretais acerca da construção de uma ferramenta tecnológica que facilite a comunicação com o enfermeiro navegador e o acesso à informação; propor a elaboração de uma ferramenta de comunicação e divulgação de conhecimento alinhadas às necessidades dos participantes da pesquisa. Método: Estudo descritivo, qualitativo, realizado com pacientes em tratamento para câncer colorretal no Centro de Oncologia de um hospital particular, de São Paulo, Brasil. Os dados foram coletados por meio de pesquisa em prontuário e entrevistas semiestruturadas, utilizando-se a Análise Qualitativa do Conteúdo como referencial metodológico. Resultados: Sugestões foram propostas pelos pacientes, para a inserção de ferramentas tecnológicas que atendam melhor às suas necessidades, como o Whatsapp Business, para viabilizar a comunicação com os enfermeiros navegadores. Foi criada, também, uma página de Instagram, para disponibilizar conteúdo científico e material educacional sobre o câncer colorretal, denominada Colorretal_News. Conclusão: A tecnologia é percebida pelos pacientes como uma estratégia facilitadora da comunicação com os profissionais de saúde, além de fonte de conhecimento sobre a doença. Acredita-se que a implementação das propostas de intervenção tecnológica do presente estudo no serviço de oncologia otimize a comunicação entre pacientes e enfermeiros navegadores e a disponibilização de informações atualizadas e confiáveis sobre as questões relacionadas aos tumores colorretais.
\end{abstract}

Palavras-chave: Enfermagem Oncológica; Navegação de Pacientes; Aplicativos Móveis; Neoplasias Colorretais; Cuidados de Enfermagem.

\section{Proposing a Technological Tool for Communication between Nurses and Patients in Oncology}

Abstract: Introduction: The presence of the navigator nurse in the oncology patient care scenarios has become a trend in Brazil and in the world, with an emphasis on colorectal cancer. One of the main approach strategies used by these professionals is communication, which has been facilitated with the use of technology. Objectives: To understand the expectations of patients with colorectal tumors regarding the construction of a technological tool that facilitates communication with the nurse navigator and access to information; propose the development of a communication and knowledge dissemination tool in line with the needs of research participants. Method: Descriptive, qualitative study conducted with patients undergoing treatment for colorectal cancer at the Oncology Center of a private hospital, in São Paulo, Brazil. Data were collected through medical record research and semi-structured interviews, using Qualitative Content Analysis as a methodological framework. Results: Suggestions were proposed by patients, for the insertion of technological tools that better meet their needs, such as Whatsapp Business, to enable communication with nurse navigators. An Instagram page was also created to provide scientific content and educational material on colorectal cancer, called Colorretal News. Conclusion: Technology is perceived by patients as a strategy to facilitate communication with health professionals, as well as a source of knowledge about the disease. It is believed that the implementation of the technological intervention proposals of the present study in the oncology service optimizes communication between patients and nurse navigators and the provision of updated and reliable information on issues related to colorectal tumors.

Keywords: Oncology Nursing; Patient Navigation; Mobile Applications; Colorectal Neoplasms; Nursing Care. 


\section{Introdução}

O enfermeiro navegador em oncologia é o profissional que possui conhecimento e habilidades avançados na área, desempenha funções de práticas avançadas, fornece assistência individualizada e possibilita ao paciente ultrapassar as barreiras dos sistemas de saúde (Oncology Nursing Society, 2017).

Além da presença do enfermeiro navegador, outra tendência no desenvolvimento da assistência à saúde é o emprego da tecnologia. A utilização de aplicativos móveis e outras ferramentas de comunicação online traz grandes avanços à prática da enfermagem (Chang, Zhao, Qiao, Yao, Wang, Li, et al, 2018; DeLemos, 2017; Chuang, Liu, Fu, Liu, Chien, Lin et al, 2015; Boston-Fleischhauer, 2017).

A tecnologia transformou o modo como nos comunicamos e acessamos informações. A utilização de aplicativos e redes sociais permite acesso instantâneo às necessidades, através de operações simples (Oncology Nursing Society. 2017; Deen, Terna, Kim, Leahy, \& Fedder, 2018). Isso é comprovado por estudos que demonstraram diminuição no tempo de acesso dos pacientes aos enfermeiros e facilidades no reconhecimento, pelo enfermeiro, do paciente em necessidade (Chang, Zhao, Qiao, Yao, Wang, Li, et al, 2018; Chuang, Liu, Fu, Liu, Chien, Lin et al, 2015).

Ainda sobre os benefícios dos avanços da tecnologia na saúde e da presença do enfermeiro navegador no atendimento aos pacientes oncológicos, destacam-se os benefícios em prol dos pacientes acometidos por tumores de cólon e reto. Esta população de pacientes está frequentemente exposta a diversas modalidades de tratamento e, muitas vezes, tem o tratamento estendido ao domicílio - no caso da quimioterapia via oral e dos infusores de quimioterapia portáteis (Manual de Oncologia Clínica do Brasil, 2018; Bonassa, \& Gato, 2012).

Diante disso, a seguinte questão norteou a realização deste estudo: "Quais seriam as sugestões dos pacientes com tumores colorretais acerca da construção de uma ferramenta tecnológica que facilitasse a comunicação com o enfermeiro navegador e o acesso à informação?".

Desta forma, este artigo tem o objetivo de: compreender as expectativas dos pacientes com tumores colorretais acerca do uso de uma ferramenta tecnológica que facilite a comunicação com o enfermeiro navegador e o acesso à informação; e propor a elaboração de uma ferramenta de comunicação e divulgação de conhecimento para enfermeiros e pacientes, conforme as necessidades dos participantes da pesquisa.

\section{Metodologia}

Trata-se de um estudo descritivo, qualitativo, realizado no Centro de Oncologia de um hospital particular de nível quaternário, do município de São Paulo, Brasil.

Dez pacientes com câncer colorretal em tratamento na referida instituição, foram convidados a participar do estudo durante a coleta de dados, entretanto quatro recusaram a participação. Assim, a amostra constituiu-se de seis pacientes, entre 47 e 77 anos de vida (média=60 anos), sendo quatro homens e duas mulheres. Quanto ao grau de instrução, Quanto ao grau de formação, três participantes possuíam nível superior, dois eram pós graduados e um possuía nível técnico. Cinco participantes possuíam tumor de cólon e um deles o tumor de reto e, em relação ao tempo decorrido desde o diagnóstico, a média foi de 18 meses, variando de um a 53 meses.

O número de participantes foi definido por inclusão progressiva, encerrando-se a coleta de dados quando as informações obtidas permitiram às pesquisadoras compreender a situação estudada. Não foram incluídos na amostra aqueles que apresentaram barreiras físicas, como impossibilidade de fala e dificuldades com o idioma e/ou cognitivas, que dificultassem a compreensão do conteúdo da entrevista. 
Os dados foram coletados em 2020 e na primeira etapa obteve-se informações sobre o perfil epidemiológico dos participantes por meio de pesquisa em prontuário eletrônico. Utilizou-se um instrumento elaborado pelas autoras, contendo informações como idade, gênero, nível de instrução, diagnóstico médico e tempo de evolução da doença.

$\mathrm{Na}$ segunda etapa, os pacientes participaram de uma entrevista semiestruturada, conduzida a partir das seguintes questões norteadoras: De que forma você considera que o uso da tecnologia pode ajudar na comunicação com o seu enfermeiro navegador? Se você tivesse acesso a um aplicativo para a comunicação com o enfermeiro navegador, em que situações você o utilizaria? Em relação ao conteúdo, o que você gostaria de encontrar nesse aplicativo?

As entrevistas foram gravadas em dispositivo de áudio, previamente autorizadas pelos participantes, e duraram em média 20 minutos, sendo realizadas individualmente em um consultório do ambulatório de oncologia e agendadas conforme a disponibilidade dos participantes.

O projeto foi avaliado pelo Sistema Gerenciador de Projeto de Pesquisa pelo Comitê de Ética em Pesquisa do Instituto de Ensino e Pesquisa do Hospital Israelita Albert Einstein, em cumprimento à Resolução 466/12, do Conselho Nacional de Saúde, tendo sido aprovado sob o parecer $n^{\circ} 3.420 .827$ (CAAE: 14469919.4.0000.0071). Após serem informados sobre a pesquisa e concordarem em participar, os pacientes assinaram o Termo de Consentimento Livre e Esclarecido.

As entrevistas foram transcritas literalmente e analisadas por meio da Análise Qualitativa do Conteúdo, um método que propõe classificações sistemáticas pela identificação de temas ou padrões, possibilitando a interpretação subjetiva do conteúdo. Amplamente utilizada na área da saúde, abrange duas fronteiras: a tradicional ou linguística e a hermenêutica, relacionada a interpretação do sentido das palavras (Franco, 2007; Campos, 2004).

Esta estratégia metodológica permite descrever um determinado fenômeno, ampliando o seu conhecimento sem a perda do rigor científico e possui três modalidades: dirigida, somatória e convencional (Franco, 2007).

A modalidade dirigida é utilizada em pesquisas iniciais ou quando há uma teoria ou pesquisa prévia sobre um fenômeno considerada incompleta ou que pode se beneficiar de um maior detalhamento (Franco, 2007; Hsieh, \& Shannon, 2005).

A modalidade somatória caracteriza-se pela criação de códigos, identificados a partir de palavras-chave, contadas e comparadas entre si, de modo que os dados são analisados pela quantidade de vezes que estas se repetem no material analisado. Como a abordagem qualitativa de análise dos dados vai além da contagem de palavras, procede-se, em seguida, a análise do conteúdo latente, buscando-se compreender o uso contextual destas palavras e descobrir seus significados (Franco, 2007; Hsieh, \& Shannon, 2005).

A modalidade convencional, utilizada no presente estudo, mostra-se apropriada quando a teoria existente relacionada à pesquisa é escassa ou limitada, privilegiando-se o uso de entrevistas não estruturadas, com perguntas abertas para a obtenção dos dados (Franco, 2007; Hsieh, \& Shannon, 2005).

Nesta modalidade, os pesquisadores evitam usar categorias pré-concebidas, de modo que é a partir do discurso dos participantes que se derivam as categorias e os códigos para análise dos dados, como ocorreu neste estudo. Para orientar este processo, um diagrama com a organização das categorias e das subcategorias em uma estrutura hierárquica pode ser empregado (Hsieh, \& Shannon, 2005).

Quatro etapas compõem a análise qualitativa de conteúdo convencional:

- Codificação dos dados, realizada por meio de leituras repetidas e menos aprofundadas do material, de modo a promover a imersão do pesquisador no conteúdo e obter um sentido do todo. São feitas marcações e anotações, respeitando-se as palavras e expressões utilizadas pelos participantes da pesquisa que devem ser mantidas, para que possam ser captadas as palavras-chave; 
- Categorização dos dados, quando são agrupados os trechos com significado semelhante ou outras similaridades, derivando as categorias e subcategorias;

- Integração das categorias, quando o pesquisador observa relações entre as categorias encontradas e analisa os significados recorrentes;

- Descrição dos dados que, após categorizados, são relacionados às anotações feitas durante o processo e ao material encontrado na literatura ou utilizado na pesquisa (Campos, 2004; Hsieh, \& Shannon, 2005).

Os dados foram analisados seguindo os passos de codificação dos dados, categorização, integração das categorias e descrição, sendo validado por pares.

Os discursos evidenciaram duas categorias temáticas: "Dando voz aos pacientes com câncer colorretal sobre o uso de tecnologia na comunicação com o profissional", constituído pelas subcategorias "Comunicação assertiva com o enfermeiro navegador", "Considerando a tecnologia como um meio de obter informações seguras" e "Simplicidade, disponibilidade, atualidade e comunicação assertiva"; e "Propondo ferramentas tecnológicas que otimizem a comunicação entre enfermeiro e paciente". Prezando pelo sigilo dos participantes, os trechos dos discursos foram identificados com a letra $E$ (entrevistado) e o número correspondente à entrevista realizada.

\section{Resultados}

\subsection{Dando Voz aos Pacientes com Câncer Colorretal sobre o Uso de Tecnologia na Comunicação com o Profissional}

\subsubsection{Comunicação Assertiva com o Enfermeiro Navegador}

A tecnologia é considerada, pelos entrevistados, uma forma atual e eficaz na comunicação com o enfermeiro navegador. Eles acreditam que as ferramentas tecnológicas podem tornar a comunicação com os profissionais quase instantânea, possibilitando a troca de informações visuais, como vídeos e fotografias.

Olha, eu acho que só vem a somar, não é mesmo? Esses Apps da vida, que permitem uma comunicação quase que instantânea (E3).

Então, eu precisei ligar para eles (os profissionais), acho que duas vezes nesse tempo todo, né? e me atenderam de pronto, né? Só que esse telefone, por algumas razões, eles não habilitaram o Whatsapp. Então, você tem que ligar mesmo, entendeu? [...] Não é tão legal quanto você poder mandar uma foto, poder mandar um vídeo... ou então, tá aqui minha temperatura... entendeu? (E2).

Sobre a ferramenta tecnológica citada por quase todos os entrevistados, o Whatsapp é reconhecido por sua eficácia, agilidade e possibilidade de condensar informações. Além disso, os pacientes consideram a troca de mensagens por Whatsapp menos invasiva.

Quando você usa o Whatsapp, até pela maneira de você se comunicar, é uma coisa mais...você concentra mais o assunto que quer colocar, e as pessoas estão mais ligadas o tempo todo com o Whatsapp. É mais imediato o contato (E1).

Então, eu acho que o mais legal, para poder falar diretamente, seria um Whatsapp. Todo mundo prefere passar texto atualmente, é menos invasivo e tudo mais (E2).

Os participantes referem que, se tivessem disponível uma ferramenta tecnológica para se comunicar com o enfermeiro navegador, a utilizariam em situações pontuais, principalmente na vigência de algum efeito colateral fora do esperado. 
Por exemplo, se surgisse alguma coisa nova da minha parte... uma anomalia não prevista, eu comunico rapidamente, né? Por exemplo, né, uma coisa que fosse fora do esperado (referindo-se a efeitos colaterais). (E3).

Uma vez ou outra, para tirar dúvidas emergenciais, para tirar dúvidas pontuais. Uma vez ou outra, só. Não há mais, tirando essas razões, demandas pra encher o saco de ninguém (E5).

Para um dos participantes, o uso da tecnologia em saúde não é livre de riscos, entendendo que o profissional nem sempre consegue documentar as informações transmitidas aos pacientes:

Então eu falo, posso te ligar? Estou com dor de barriga... Então, eu ligo no ramal. Não é gravado, né? Você vai ter que se virar nos 30, daí. Então, é assim, você tem soluções de comunicação e se você não tem tudo documentado, o cara te põe no pau dois anos depois. Você que tem que ter as evidências de que o serviço foi certo. [...] Então, tem que tomar muito cuidado, prestar muita atenção. É muita informação contida ali, né? (E5).

\subsubsection{Considerando a Tecnologia como um Meio de Obter Informações Seguras}

A busca de informações por meio de conteúdo digital de confiança pareceu ser uma grande preocupação para os entrevistados. Os relatos dos pacientes apontam para o interesse em procurar informações que pareçam fidedignas e sérias junto a sites científicos.

De uma forma geral estou muito bem informado, em literatura científica. Então, são "papers", são estudos, coisas serias. Ou seja, estudos que tem toda parte estatística. Eu sou matemático, cientista da computação. Então, toda parte estatística, eu posso ver a seriedade dos estudos que são feitos... (E2).

Sensacional! Eu obtinha todas as respostas. Eu sempre podia procurar pelas dúvidas que eu tinha. Recebia todos os materiais e sempre tudo muito completo, de fontes. Porque nem tudo que "tá" online é verdade, então tem muitas fontes fidedignas. (E5).

Ainda em relação à fidedignidade das informações, alguns pacientes preferem não utilizar a internet para esse fim, preferindo confiar nas informações oferecidas pelo médico.

Não! Para mim é que nem o médico fala: agora, você vai ter que confiar em mim. Você tem que confiar na minha palavra. Então, não procuro (informações na internet) por não querer mesmo. Porque quanto mais você procura, muitas vezes, você vai interpretar uma coisa que é "desse tamaninho" (gesticula com as mãos referindo-se a algo pequeno) e é "desse tamanho" (gesticula com as mãos referindo-se a algo grande). (E4).

\subsubsection{Simplicidade, Disponibilidade, Atualidade e Comunicação Assertiva}

Em relação à disponibilização de conteúdo, os participantes acreditam que o emprego de ferramentas tecnológicas na comunicação e na troca de informações é muito relevante, não apenas para o paciente, mas também para o profissional de saúde.

Não (se utiliza a tecnologia para obter informações), mas se tivesse alguma tecnologia, sempre é melhor! A tecnologia é sempre para facilitar para o paciente e facilitar, também, para a pessoa que está trabalhando, os enfermeiros. (E6). 
Os discursos evidenciam também que para os participantes do estudo, o conteúdo abordado na ferramenta tecnológica deveria contemplar esclarecimentos e informações sobre as patologias oncológicas, seu estadiamento e o tratamento proposto, além das novas possibilidades e avanços científicos na área.

Então, para cada um desses tipos de tumor e tipo de estadiamento, você vai ser submetido a um tipo de medicação mais agressivo ou menos agressivo... Então, podia ter um tipo de um FAQ (Perguntas mais frequentes). Podia ser assim: O cara tem câncer colorretal, estádio III, os tratamentos vão ser oxaliplatina, não sei o que... Então, estão aqui as 10 ou 15 perguntas mais frequentes. [...] (E2).

Outra questão destacada pelos entrevistados é barreira que a linguagem científica pode trazer à compreensão, além da limitação idiomática. Desta forma, sugerem o uso de uma linguagem simplificada que facilite a compreensão dos pacientes na elaboração da ferramenta.

Acho que poderia ter um resumo de tudo, né? Porque, por exemplo, nem todos os pacientes falam inglês, nem todos entendem a sopa de letras, sopa de siglas. Então, eventualmente, com a estatística também...então, não sabem o que é um hazard ratio, intervalo de confiança. Não sabe nada disso, né? Simplifica para o paciente... Para tirar esse viés científico e tudo... E vocês, que estão focados nisso, vão achar esse conteúdo o tempo todo. (E2).

Os relatos destacam, também, questões relacionadas à aplicabilidade da ferramenta, no sentido de manter atualizado o conteúdo. Torna-se clara a preocupação com a manutenibilidade da ferramenta, a fim de que não deixe de ser interessante.

Aí, vira uma enciclopédia. Só isso! E nada que não vá ser manutenível. A pior coisa que tem é você fazer a coisa mais legal do mundo e depois não conseguir manter atualizado. É mais prejudicial do que não fazer. (E5)

Olha, informação sempre é interessante, mas eu vejo muito pela revista do (cita o nome do laboratório). Antes, era mensal mas, cara, não tem informação suficiente pra você atualizar uma revista com conteúdo de qualidade mensal! Então, eu acho que tem que fazer uma atualização bimestral, trimestral ou quando algo importante e relevante surgir e ser cumulativo. (E2).

\subsection{Propondo Ferramentas Tecnológicas que Otimizem Comunicação entre Enfermeiro e Paciente}

A partir das sugestões contidas nos discursos dos pacientes, considerou-se o uso do Whatsapp Business, que permite comunicação instantânea, troca de mensagens de texto e de voz, além do envio de documentos (Whatsapp, 2020). Ressalta-se, ainda, que o aplicativo permite o envio de mensagens automáticas, recurso que poderia ser utilizado para informar os pacientes sobre o horário de funcionamento do serviço.

Em relação à troca de informações entre enfermeiros e pacientes, foi criada uma página no Instragram, direcionada àqueles com tumores colorretais. O Instagram é uma ferramenta para criação e compartilhamento de conteúdo e, assim como o Whatsapp, tem a opção da construção de perfil business (Instagram, 2020), opção escolhida para a criação da referida página.

A página, intitulada "Colorretal News", com usuário "colorretal_news", foi inserida na categoria "saúde/beleza". No momento, a página encontra-se em criação de conteúdo. As informações atualizadas sobre câncer colorretal estão sendo publicadas com a inclusão de imagens e links de acesso à referência do periódico ou local de onde foram extraídas. Os recursos de publicação de vídeos e do Instagram Stories também serão utilizados, com a participação de especialistas. Abaixo de cada publicação, será disponibilizado um resumo simplificado a respeito do conteúdo. 
Ressalta-se que todas as informações disponibilizadas nessa ferramenta terão rigoroso embasamento científico, sendo validadas por um segundo enfermeiro e um médico oncologista, antes da sua publicação.

\section{Discussão}

Em relação à primeira categoria, "Comunicação assertiva com o enfermeiro navegador", apesar de reconhecerem a eficácia das ferramentas tecnológicas na comunicação com o profissional, atualmente, os pacientes contam com a comunicação por meio de e-mail e de ligações telefônicas, disponibilizadas pela instituição. Aqueles que se comunicam com o enfermeiro por meio de Whatsapp, o fazem pelo aplicativo pessoal do profissional.

A atuação do enfermeiro navegador mediada por ligações telefônicas é uma prática ainda pouco explorada na literatura, porém pode ser observada em outros serviços e aparenta trazer benefícios aos pacientes, como maior adesão aos tratamentos e menos faltas às consultas e aos procedimentos (Johnson, 2016).

Segundo os participantes do presente estudo, eles utilizam as ferramentas tecnológicas para buscar informações sobre a doença e consideram que a tecnologia é o meio mais eficaz de se comunicar com o enfermeiro navegador e obter informações individualizadas. No tocante à comunicação, a principal ferramenta abordada pelos participantes foi o Whatsapp.

Além disso, os entrevistados demonstraram preocupação com a fidedignidade das informações encontradas no acesso à internet e consideraram o enfermeiro navegador como um profissional capaz de transmiti-las com segurança aos pacientes.

Dados de um ensaio clínico randomizado demonstraram ganhos de eficácia na transmissão de informações online a pacientes e na implementação da navegação por pares. Os pacientes que receberam as intervenções apresentaram melhores índices de conhecimento e eficácia na realização dos cuidado (Casillas, Schwartz, Crespi, Ganz, Kahn, Stuber, et al, 2019).

A literatura reforça, portanto, que, por meio da disponibilização de informações online, o enfermeiro tem possibilidade de contribuir para a maior autonomia do paciente e sua conscientização, principalmente nos cuidados preventivos (Denecke, Kyburz, Gfeller, Deng, \& Bürkle, 2018)

Porém, em contrapartida, um trabalho que buscou avaliar a opinião dos pacientes sobre um aplicativo móvel de comunicação com o enfermeiro navegador demonstrou que, em alguns casos, o interesse pelo seu uso não foi significativo. As razões para esta atitude relacionaram-se principalmente ao meio que a ferramenta foi disponibilizada, ao cansaço do cotidiano e à falta de tempo (Gehrke, Lee, Hilton, Ganster, Trupp, McCullough, et al, 2018).

Um dos participantes da presente pesquisa demonstrou preocupação sobre a proteção dos dados na troca de informações com os pacientes. Segundo ele, os dados transmitidos por ligações telefônicas muitas vezes não são gravados, podendo trazer implicações futuras para o profissional, uma vez que não há como registrar as orientações oferecidas.

Em concordância com o discurso deste paciente, apesar de ser uma tendência global o uso de dispositivos móveis na saúde representa algumas preocupações sobre regulamentação e vigilância. Assim, em 2018, o Conselho Federal de Medicina publicou a Resolução no 2178/2018, para oferecer apoio e segurança aos pacientes e profissionais que utilizam este tipo de atendimento, no caso, os aplicativos que oferecem consulta médica em domicílio (Conselho Federal de Medicina, 2018).

Já, em relação à segunda categoria do presente estudo, "Simplicidade, disponibilidade, atualidade e comunicação assertiva...", os participantes enfatizaram a importância de se privilegiar conteúdos mais atualizados e individualizados. Sugeriram, também, a disponibilização de um "FAQ" que contemplasse as dúvidas mais frequentes dos pacientes sobre determinado assunto. 
A respeito do conteúdo, os participantes também destacaram a importância de que a ferramenta seja manutenível. A literatura corrobora esta observação dos pacientes, uma vez que há relatos sobre o risco do excesso de informações que este tipo de ferramenta pode trazer, o que faz com que não seja utilizada ou não pareça relevante (Gehrke, Lee, Hilton, Ganster, Trupp, McCullough, et al, 2018).

Assim, em relação à terceira categoria, "A criação da ferramenta tecnológica", o Whatsapp web foi proposto para mediar a comunicação entre pacientes e enfermeiros navegadores, bem como uma conta de Instagram, para disponibilização de conteúdo, denominada "colorretal_news", levando-se em consideração as necessidades, as sugestões e os desejos dos participantes do estudo.

Por fim, a literatura mostra que o uso das ferramentas tecnológicas no trabalho da enfermagem pode constituir-se em mais uma ferramenta efetiva para o atendimento, com o benefício de conceder ao paciente maior autonomia para administrar suas condições crônicas de saúde (Samples, Zhao, \& Shaw, 2014).

\section{Conclusões}

Os pacientes demonstraram grande interesse pela comunicação com os profissionais e pela busca de informações online sobre seu estado de saúde, preocupando-se com a fidedignidade e a facilidade de compreensão das informações, bem como acesso a dados atuais e individualizados.

Dentre as sugestões propostas para a construção de uma ferramenta tecnológica que viabilize a comunicação com a equipe do setor de oncologia, o uso do Whatsapp Busines mostrou-se mais apropriado para esse fim. E para atender às necessidades de informação científica sobre a doença, foi criada uma página no Instagram, dedicada à divulgação de conteúdos relacionados aos tumores colorretais.

Considerando os achados do estudo, sugere-se a realização de novos estudos com pacientes atendidos na rede pública de saúde e em outras regiões do Brasil, tendo em vista as discrepâncias na qualidade do atendimento recebido e as limitações de acesso aos meios tecnológicos por questões geográficas e socioeconômicas.

A investigação qualitativa traz significativas contribuições para estudos deste tipo em que se busca dar voz aos usuários de produtos resultantes de pesquisa para atender às suas demandas cotidianas, como ferramentas tecnológicas entre outros, de modo a alinhá-los às reais necessidades da clientela que o utilizará.

\section{Referências}

Bonassa, E.M., \& Gato, M.I. (2012). Terapêutica oncológica para enfermeiros e farmacêuticos. (4a ed.) São Paulo: Atheneu.

Boston-Fleischhauer, C. (2017). The Explosion of Virtual Nursing Care. J Nurs Adm.,47(2):85-7.

Campos, C.J. (2004). Método de análise de conteúdo: ferramenta para a análise de dados qualitativos no campo da saúde. Rev Bras Enferm., 57(5):611-4.

Casillas, J.N., Schwartz, L.F., Crespi, C.M., Ganz, P.A., Kahn, K.L., Stuber, M.L, et al. (2019). The use of mobile technology and peer navigation to promote adolescent and young adult (AYA) cancer survivorship care: results of a randomized controlled trial. J Cancer Surviv.,13(4):58092.

Chang, H., Zhao, J., Qiao, Y., Yao, H., Wang, X., Li, J, et al. (2018). Mobile phone application for self-assessment of acute stroke patients: A tool for extended care and follow-up. Medicine.,97(26):e11263.

Chuang, S.T., Liu, YF, Fu ZX, Liu KC, Chien SH, Lin CL, et al. (2015). Application of a smartphone nurse call system for nursing care. Telemed J E Health.,21(2),105-9. 
Vol. 8 | Investigação Qualitativa em Saúde: Avanços e Desafios

Conselho Federal de Medicina. (2018). Resolução CFM. n. 2.178/2017, de 14 de dezembro de 2017. Regulamenta o funcionamento de aplicativos que oferecem consulta médica em domicílio. Diário Oficial da União.; Seção 1:138.

Deen, T., Terna, T, Kim, E., Leahy B, \& Fedder W. (2018). The impact of stroke nurse navigation on patient compliance post discharge. Rehabil Nurs, 43(2): 65-72.

DeLemos, C.D. (2017). Developing a Mobile App for Neuroscience Nurses. J Nurs Adm.,47(9): 413-4.

Denecke, K., Kyburz, P, Gfeller, S, Deng, Y,\& Bürkle,T. (2018). A Concept for Improving CrossSector Care by a Mobile Patient Navigator App. Stud Health Technol Inform.,255: 160-4.

Franco, M.L. (2007). Análise de conteúdo (2a ed.). Brasília (DF): Líber Livro.

Gehrke, A., Lee, S.S, Hilton, K., Ganster, B, Trupp, R., McCullough, C., et al. (2018). Development of the Cancer Survivor Profile-Breast Cancer (CSPro-BC) app: patient and nurse perspectives on a new navigation tool. J Cancer Surviv.,12(3), 291-305.

Hsieh, H.F.,\& Shannon, S.E. (2005). Three approaches to qualitative content analysis. Qual Health Res.,15(9),1277-88.

Instagram.(2020). Sobre o instagram. https://about.instagram.com/

Johnson, F. (2016). The Process of Oncology Nurse Practitioner Patient Navigation: A Pilot Study. Clin J Oncol Nurs., 20(2), 207-10.

Manual de Oncologia Clínica do Brasil. (2018). Câncer gastrintestinal: cólon. https://mocbrasil.com

Oncology Nursing Society. (2017). Oncology Nurse Navigator Core Competencies. https://www.ons.org/sites/default/files/201705/2017_Oncology_Nurse_Navigator_Competenci es.pdf

Samples, C., Zhao, N,,\& Shaw, R.J.(2014). Nursing and mHealth. Int J Nurs Sci,1(4), 330-3.

Whatsapp. (2020). Aplicativo Whatsapp business. https://www.whatsapp.com/business/?lang=pt_br 